Itinéraires Itinéraires

Littérature, textes, cultures

\title{
Régine Robin, Mégapolis. Les derniers pas du flâneur
}

Paris, Stock, coll. « Un ordre d'idées », 2009, 397 p.

\section{François Masse}

\section{CpenEdition}

Journals

Édition électronique

URL : http://journals.openedition.org/itineraires/2137

DOI : 10.4000/itineraires. 2137

ISSN : 2427-920X

Éditeur

Pléiade

\section{Édition imprimée}

Date de publication : 1 mai 2010

Pagination : 178-184

ISBN : 978-2-296-11224-7

ISSN : 2100-1340

Référence électronique

François Masse, «Régine Robin, Mégapolis. Les derniers pas du flâneur », Itinéraires [En ligne], 2010-1 I

2010, mis en ligne le 01 mai 2010, consulté le 22 septembre 2020. URL : http://

journals.openedition.org/itineraires/2137 ; DOI : https://doi.org/10.4000/itineraires.2137

Ce document a été généré automatiquement le 22 septembre 2020.

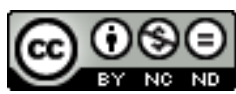

Itinéraires est mis à disposition selon les termes de la licence Creative Commons Attribution - Pas d'Utilisation Commerciale - Pas de Modification 4.0 International 


\section{Régine Robin, Mégapolis. Les derniers pas du flâneur}

Paris, Stock, coll. « Un ordre d'idées », 2009, 397 p.

François Masse

\section{RÉFÉRENCE}

Régine Robin, Mégapolis. Les derniers pas du flâneur, Paris, Stock, coll. « Un ordre

d'idées ", 2009, 397 p.

1 Née à Paris de parents juifs-polonais et ayant immigré à Montréal au milieu des années soixante-dix, Régine Robin poursuit à travers des essais, des romans et des ouvrages de théorie une réflexion sur l'identité et la parole "migrantes» (La Québécoite, 1983, Cybermigrances, 2004), sur la mémoire (Le Roman mémoriel, 1989), ou encore sur le devenir des grandes villes à l'heure de leur transformation accélérée (Berlin Chantiers, 2001). Dans son dernier ouvrage intitulé Mégapolis. Les derniers pas du flâneur, elle s'intéresse au flâneur, cette figure de la modernité avec laquelle nous a familiarisé Walter Benjamin (à travers l'œuvre de Baudelaire surtout, mais aussi par sa propre expérience de citadin), et se demande si celuici a encore une place dans les mégapoles, ces villes monstrueuses qui, si elles n'interdisent pas la flânerie, la rendent cependant de plus en plus périlleuse.

\section{Du flâneur traditionnel aux « nouvelles postures »}

2 Car, écrit Régine Robin, «ce n'est pas seulement le tissu urbain qui a changé, mais les conditions de possibilité de la flânerie et de sa mémoire » (p. 89). Le flâneur se tenait au "seuil de deux mondes [...], seuil de la rue dans les passages qui étaient son monde, seuil de la société reconnue et de la bohème, seuil de deux époques, de deux cultures » (p. 84). Or, les deux conflits mondiaux, la chute des grandes idéologies et le développement croissant des villes au $\mathrm{xx}^{\mathrm{e}}$ siècle nous ont considérablement éloignés du 
monde dont, même en son temps, le flâneur baudelairien se sentait à peine contemporain. Rappelons-nous ces vers du Cygne de Baudelaire :

Le vieux Paris n'est plus (la forme d'une ville

Change plus vite, hélas! que le cœur d'un mortel) ${ }^{1}$

De là, deux avenues possibles pour le flâneur d'aujourd'hui : persister dans la voie de Baudelaire et de Benjamin, c'est-à-dire flâner "à l'ancienne », dans le souvenir de ce Paris idéalisé où, en dépit de l'étrangeté de la foule et des dangers inhérents à la ville, le flâneur pouvait se sentir partout chez lui² ; ou bien voir s'il n'y aurait pas «une nouvelle posture à trouver» (p.88), comme le fait Régine Robin en soupçonnant que c'est le mot même de flâneur qui ne s'accorde pas à la réalité des mégapoles. L'auteure de Mégapolis présente ainsi une série de portraits d'artistes - écrivains, photographes, cinéastes et performeurs - qui, chacun à leur façon, ont tenté de "réinventer la déambulation, la traversée des métropoles, pour transformer notre regard, notre rapport à la ville, pour piéger nos habitudes, nos horaires, nos parcours obligés, pour faire que nous puissions simplement y trouver une place sans nous y installer » (p. 89).

Qu'il s'agisse du groupe italien Stalker s'aventurant dans les terrains vagues et les zones les plus désaffectées de Rome, ou de Laurent Malone et Dennis Adams effectuant en ligne droite la distance qui sépare le métro Delancey, situé dans le Lower East Side de Manhattan, de l'aéroport JFK, ces artistes rompent bel et bien avec le modèle du flâneur tel qu'il perdura de Baudelaire aux situationnistes, en passant par les surréalistes, dont les dérives «[étaient] des résistances à la normalisation, à l'uniformisation consécutives aux refontes urbanistiques». Qui plus est, d'ajouter Régine Robin, ces dérives se situaient toutes, «sauf exception, au cœur de Paris, d'un Paris à l'abri de ces gigantesques remaniements » (p.103). On comprend dès lors pourquoi Paris ne peut figurer parmi les mégapoles choisies par Régine Robin pour ses traversées. Outre le fait qu'il s'agit de sa ville d'origine, qu'elle y a « des souvenirs à chaque carrefour, à chaque station de métro, à chaque arrêt d'autobus » (p. 23), celle-ci n'apparait pas comme le terrain le plus propice pour expérimenter ces «nouvelles postures » de la flânerie que sont par exemple le nomade, l'automobiliste ou le « cruiser $^{3}$ ».

Or si, dans ses pérégrinations urbaines, Régine Robin n'hésite pas à adopter ces nouvelles postures de la flânerie, elle demeure néanmoins attachée à la figure traditionnelle du flâneur; elle ne veut pas renoncer non plus à ce terme, se qualifiant elle-même de "dernière flâneuse » (p. 150), de "flâneur sociologique » (p. 21). De même, on pourrait dire qu'elle demeure prise entre les deux attitudes qui caractérisent le flâneur, attitudes opposées, mais qui se chevauchent à l'intérieur de lui : soit le désir de mobilité incessante, celui qui fait du flâneur un être en état de partance continuel et, simultanément et paradoxalement, le désir de se fixer quelque part, de faire de la rue un endroit où élire domicile. Car, nous rappelle Régine Robin, c'est un fait que le flâneur, loin de se sentir désorienté, d'être en mal de repères dans la ville, est, au contraire, celui qui est aux prises avec un environnement trop familier : «La rue est son lieu d'élection. Il est chez lui quoi qu'il en dise. Ce qu'il cherche dans la ville où il a ses repères, c'est précisément à leur échapper, à se sentir autre, à s'altérer. Il est en quête de l'incongru, de ce qui est en marge, de ce qui vient rompre la familiarité des lieux» (p. 101). Tout à l'opposé est le nomade ou le "cruiser», qui est par nature désorienté, sans cesse occupé à déchiffrer la signalisation, à tenter de se repérer dans des espaces infiniment redondants - les autoroutes, les motels, les stationnements -, des espaces qui sont certes toujours les mêmes, mais qu'il lui faut néanmoins toujours 
réapprivoiser. Le nomade est un "analphabète urbain» (p. 102), "il doit en permanence transformer l'inconnu en quelque chose de familier. C'est lui qui est en état d'hypnose, jamais le flâneur » (p. 102). En ce sens, ajoute Robin, « [le nomade] est plongé dans une opération que le flâneur pourrait lui envier » (p. 101).

6 Revenir au principe fondamental de la flânerie, à l'errance pure et simple, tel est donc le programme que se propose Régine Robin dans ses traversées urbaines qui, effectivement, ne comportent presque pas de rencontres et où peu de haltes sont effectuées. Ce sont, à proprement parler, des traversées. D'ailleurs, la plupart des films et des romans évoqués par Robin dans son ouvrage ne le sont pas tant en raison de leur sujet ou de leur valeur esthétique, mais parce qu'ils mettent en scène la traversée de la ville. Il en est ainsi de Collateral de Michael Mann, ou des romans policiers de Michael Connelly par exemple. Or, malgré sa volonté de s'en tenir à la traversée brute, à la déambulation vécue pour elle-même, la flâneuse postmoderne ne peut lutter contre le magnétisme des choses qui nous invitent à nous attacher à elles. Car comment résister à la tentation de s'ancrer au sein des mégapoles, quand on y a vécu au quotidien et après en avoir épousé le rythme? C'est ainsi qu'à la veille de quitter New York, Robin en a déjà la nostalgie: "Néanmoins, la panique à l'heure de quitter New York. Cette ville allait continuer sans moi»(p.114). Ce n'est donc pas contre l'inquiétante étrangeté que la flâneuse des mégapoles se trouve à lutter, mais contre l'inquiétante familiarité qui, dans l'ouvrage de Régine Robin, s'incarne dans une figure bien singulière : la petite femme de Michael Snow. De quoi s'agit-il ? D'une silhouette de femme d'un mètre cinquante-deux de haut que l'artiste canadien Michael Snow a découpée dans du carton et qu'il a placée à divers endroits dans le monde, des bouches de métro new-yorkaises jusque dans la forêt amazonienne ${ }^{4}$. Cette silhouette de femme qui va toujours, qui est sereine dans son mouvement et dans son anonymat, accompagne Régine Robin tout au long de son odyssée à travers les mégapoles.

\section{La petite femme de Michael Snow}

7 La première apparition de cette silhouette énigmatique a lieu au moment où, arpentant Broadway de haut en bas, la flâneuse se plaît à imaginer la vie des gens qu'elle observe :

Je faisais collection d'histoires de vie dépareillées. J'imaginais une biographie pour chaque femme, chaque homme que je rencontrais dans le métro, dans la rue, sur le terre-plein au milieu de Broadway, au cinéma. J'en avais des milliers, petits fragments de vie, éclats fugitifs d'existence, milliers d'itinéraires, de parcours de vies blessées et dépenaillées, à commencer par la mienne, qui tissaient autour de moi un immense et douloureux patchwork. (p. 118)

Ce phénomène qui consiste pour le flâneur à disposer de son individualité comme d'un rien, à s'identifier au sein de la foule à une multitude d'existences, et ce, dans une relation qui s'apparente à celle qui existe entre le consommateur et la marchandise d'où le fait, dit Benjamin, que "l'homme-sandwich est la dernière incarnation du flâneur ${ }^{5} »$; c'est l'homme devenu la marchandise qu'il vend-, Walter Benjamin l'appelle "l'ivresse de l'identification ${ }^{6}$ ». En vraie flâneuse, Régine Robin est prise de cette ivresse, la mégapole étant pour elle le lieu où inventer l'existence des autres, mais également et surtout le lieu où réinventer sa propre existence, se rêver autre, se démultiplier en autant de petites femmes de Michael Snow. Car c'est bien l'ivresse de l'identification que cette dernière incarne : elle est une silhouette vacante sur laquelle la flâneuse est libre de coller n'importe quel visage. Toutefois, la démultiplication de soi 
dissimule un danger : celui de se retrouver à un moment englouti par la foule, de succomber sous le poids des identités multiples au point de perdre la sienne propre. C'est ainsi qu'en proie aux apparitions de la silhouette, Régine Robin se demande : «[...] étais-je démultipliée, sans ancrage, sans assise ? Un mécanisme me faisait-il apparaître parce qu'en réalité j'avais disparu? » (p. 118-119).

9 À mesure que nous avançons dans l'ouvrage de Régine Robin, cette silhouette de femme prend de plus en plus de consistance. Celle qui ne faisait qu'apparaitre fugitivement à New York et à Los Angeles donne ainsi un rendez-vous à l'auteure à Tokyo (rendez-vous auquel cette dernière se garde bien de se rendre d'ailleurs). Mais c'est dans la partie sur Buenos Aires que la silhouette va prendre le plus d'ampleur, en s'incarnant sous les traits d'un inconnu qui, par courrier électronique, écrit à l'auteure pour lui dire qu'il souhaite lui remettre des informations sur un grand-oncle dont elle aurait oublié l'existence. Dès lors, un petit récit commence ; une histoire dans laquelle nous n'avons plus tant affaire à Régine Robin auteure de Mégapolis qu'à son homologue fictif. L'alter ego de notre auteure repart donc à Buenos Aires sur les traces de ce grand-oncle au lourd passé. En effet, durant les années 1930, celui-ci se serait commis au sein d'un réseau de prostitution, réseau basé à Buenos Aires et alimenté par des filles de Pologne et de Russie, avant de s'en retourner en Pologne pour connaître le sort réservé aux juifs : «Et puis, comme tous les autres de Mińsk Mazowiecki, il a fini à Treblinka. Les nazis ne différenciaient pas les criminels des autres » (p. 315), explique à l'auteure une femme qui se prétend être la petite-fille de Jaime Tiempo. Cette recherche se solde donc par un échec, l'inconnu entré en contact avec Robin sur Internet s'étant volatilisé et la tombe de ce prétendu grand-oncle proxénète et inventeur de tangos célèbres demeurant introuvable. "Si ce grand oncle n'avait jamais existé ?", se demande alors Robin. «À force de flâner sur l'écran, de m'inventer de nombreuses identités, j'avais peut-être fini par m'inventer un passé, un roman familial dans une ville qui était le sosie de Buenos Aires, mais qui était irréelle» (p.316). Au terme de cette partie, nous ne savons plus qui, de la silhouette de Michael Snow ou de notre flâneuse, est la poursuivante et la poursuivie. Les deux se donnent la chasse dans un parcours où le réel et l'imaginaire se confondent.

10 C'est généralement à la fin de chacune des parties de l'ouvrage que la petite femme de Michael Snow refait surface, c'est-à-dire au moment où, après avoir percé le mystère d'une mégapole et surmonté ses clichés, la flâneuse s'y sent pour ainsi dire chez-elle, complètement acclimatée. À Los Angeles par exemple, alors qu'elle est coincée dans un bouchon de circulation, presque contente de l'immobilité confortable que lui procure cette situation, l'auteure voit resurgir la silhouette dont les traits se confondent désormais avec les siens :

[...] je m'aperçois avec stupeur que toutes les conductrices ont ma tête. Il en est de même de la file de gauche et encore au-delà. C'est sans doute la raison pour laquelle nous sommes ainsi bloquées. C'est comme un virus informatique. La petite femme de Michael Snow me souffle à l'oreille, sans que je sache d'où provient sa voix : «On n'échappe pas à LA, on s'y perd, on s'y répand, on s'y démultiplie. C'est comme quand on se regarde dans certains miroirs, on se voit à l'infini. » (p. 217-218)

11 Voilà que l'ivresse de l'identification atteint son point limite, au moment où la flâneuse ne voit plus autour d'elle que des copies d'elle-même. La voix de la petite femme de Snow sonne alors comme un avertissement ; elle vient dire à la flâneuse qu'il est temps de continuer ses pérégrinations ailleurs, si elle veut échapper une nouvelle fois à l'installation et à l'identité fixée une fois pour toutes. 


\section{Les non-lieux, l'entre-deux} toutes et la démultiplication de soi, tel est le dessein que se propose Régine Robin dans ses pérégrinations urbaines. À cet égard, il faut voir comment les "non-lieux" s'avèrent servir admirablement ce dessein. Marc Augé décrit ceux-ci comme étant « aussi bien les installations nécessaires à la circulation accélérée des personnes et des biens (voies rapides, échangeurs, aéroports) que les moyens de transport eux-mêmes ou les grands centres commerciaux [...] $]^{7} »$. Parce qu'ils ont été conçus pour qu'on y entre et qu'on en sorte, autrement dit, pour qu'on n'y fasse que circuler, les non-lieux ne permettraient la formation d'aucune identité, par opposition au « lieu » qui renvoie à un endroit aux références sociales et culturelles stables, c'est-à-dire déjà fixées par une histoire et des traditions (telle la place publique, le marché ou le parvis d'église par exemple). Or, Régine Robin est loin de s'en tenir à cet usage des non-lieux, c'est-à-dire de les considérer simplement comme des espaces de transit. C'est ainsi que les moyens de transport, les gares et les centres commerciaux jouent un rôle prépondérant dans le dessein qu'elle se propose au début de son ouvrage: traquer «la poétique des mégapoles » (p. 19). Elle les investit de fond en comble et non sans un certain plaisir. Du «Bluewater», gigantesque centre commercial situé en bordure de Londres, Robin écrit :

J'ai monté et descendu cent fois les escalators. Je dois figurer mille fois sur les caméras de surveillance. Je suis entrée dans les librairies, les maroquineries, les boutiques de dessous féminins. [...] Mais cela ne m'a pas attristée. [...] J'incarne peut-être, à ma façon, ce nouveau flâneur des gigantesques centres commerciaux et des cafés qu'on retrouve partout à la surface du globe : Starbucks, Second cup, Neos, etc. (p. 346)

De même, à propos de la nouvelle gare de Kyoto, sommet d'architecture technologique, Robin dira :

Je ne pouvais détacher mon regard de cette gare, je m'y sentais bien, j'aurais pu y rester éternellement. D'ailleurs, constatant qu'un hôtel y avait été construit, le Granvia, j'ai immédiatement déménagé à cet endroit pour les quatre nuits que je devais passer à Kyoto, colonisant l'établissement, la gare, ses plis et replis, ses carrefours et bifurcations à toute heure du jour et de la nuit. (p. 225)

Qu'il s'agisse des couloirs du métro de Tokyo ou du centre commercial de Londres, Robin montre que les non-lieux sont bel et bien propices à la flânerie et, plus encore, que ce sont des lieux habitables. À la fois espaces de transit et espaces habitables, les non-lieux réalisent ainsi peut-être le compromis souhaité par Régine Robin entre l'identité fixée une fois pour toutes et la démultiplication de soi.

Les mégapoles que Régine Robin affectionne par-dessus tout dans son parcours sont celles qui, à l'instar des non-lieux, la laissent libre de s'installer tout autant que de se perdre, bref, des mégapoles où l'identité est libre de flotter :

Dans les villes patchworks, dites sans cachet comme Los Angeles, comme Montréal, dans ces juxtapositions de "villages» faits de bric et de broc, je m'épanouis, je m'insinue dans les interstices, je crée mon espace. Ce sont des villes « entre ». Elles ne me terrorisent pas par leur passé, leur monumentalité, leur patrimoine à préserver, leur urbanisme harmonieux. Je suis à l'unisson des villes déglinguées, des friches où le sens est en déroute, espaces nomades où je peux tailler ma place. (p. 224) 
Telle est également Londres qui, si elle représente l'exemple-type de la ville que ses transformations successives ont rendue monstrueuse, de la ville dont les contours se sont désagrégés dans l'étalement urbain, avec l'explosion de la banlieue, est en même temps la "ville ouverte " par excellence, celle où l'identité est libre de se réinventer à tout moment. Ainsi, Régine Robin de conclure son ouvrage en disant :

N'est-ce pas le destin des mégapoles de permettre aux identités semidétachées, diasporiques, fluides, déterritorialisées, de se développer? Londres ne le préfiguret-il pas depuis toujours? Être ou ne pas être chez soi, avoir ou ne pas avoir la nationalité du lieu, maîtriser ou ne pas maîtriser la langue de la mégapole, repartir dans son pays d'origine pour s'apercevoir qu'il faut revenir à Londres [...], rester dans l'entre-deux, cultiver la messthetics dont parle Suhdev Sandhu, habiter l'interstice. (p. 369)

«Et pourquoi pas Montréal ?» (p. 372), se demande Régine Robin en terminant. Entre le désir d'être toujours ailleurs et celui de se fixer quelque part, entre les mégapoles qu'elle a visitées hier et celles qu'elle se garde pour demain, Régine Robin alias la petite femme de Michael Snow est une flâneuse qui ne nous semble pas arrivée à ses derniers pas.

\section{NOTES}

1. Charles Baudelaire, «Le Cygne », Les Fleurs du mal, Paris, Librairie Générale Française, 1999, p. 136.

2. En effet, nous dit Walter Benjamin, le flâneur se promenait dans la ville-lumière comme au sein d'un domicile bourgeois, d'un «intérieur » entièrement meublé : «Les murs avec "Défense d'afficher" lui servent de pupitre pour écrire ; les kiosques à journaux sont ses bibliothèques, les boîtes aux lettres ses bronzes, les bancs son mobilier de chambre à coucher et la terrasse de café la fenêtre en encorbellement d'où il peut surveiller son ménage. » Walter Benjamin, Paris, capitale du xIXe siècle. Le Livre des Passages, trad. de l'allemand par Jean Lacoste, Paris, Cerf, 1989, p. 442.

3. Régine Robin emprunte cette expression, de même que son concept, le " cruising ", à Bruce Bégout qui s'en sert pour parler de l'errance en automobile, de la déambulation propre à celui qui fréquente inlassablement les autoroutes et les motels américains. Voir l'ouvrage de Bégout intitulé L'Éblouissement des bords de route, Paris, Verticales, 2004.

4. Voir Michael Snow, Biography of the Walking Woman. Biographie de la femme qui marche, Bruxelles, La Lettre volée, 2004.

5. Walter Benjamin, op. cit., p. 468.

6. Ibid., p. 466.

7. Marc Augé, Non-lieux. Introduction à une anthropologie de la surmodernité, Paris, Seuil, 1992, p. 48. 


\section{AUTEURS}

FRANÇOIS MASSE

Université McGill, Montréal 